\title{
Ubiquitous High Speed Ejecta in PNe - MyCn 18
}

\author{
J. Meaburn, M. Bryce, D. J. Harman \\ Jodrell Bank Observatory, Department of Physics and Astronomy, \\ University of Manchester, Macclesfield, Cheshire, SK11 9DL
}

\section{J. A. López}

Instituto de Astronomía, UNAM, Km 103 Carretera Tijuana-Ensenada, 22860, B.C., Mexico

Supersonic and even hypersonic outflows have been found in a wide variety of PNe using the Manchester Echelle spectrometer (MES; Meaburn et al. 1984). Dramatic examples of these extreme motions found over many years are highlighted here for comparison with their most recent discovery in the bipolar PN, MyCn 18.

The bipolar PN, MyCn 18, the Engraved Hourglass Nebula (Bryce et al. 1997; O'Connor et al. 2000), was observed using the MES at the Anglo Australian Telescope (AAT). Measurements of the radial velocities of 14 knots concluded that the radial velocities outwards from the main nebula are proportional to the distance that the knots had traveled. Outflowing velocities of $\gtrsim 630 \mathrm{~km}$ $\mathrm{s}^{-1}$ were measured for these compact knots. The interaction of a recurrent novalike ejection from a central binary system is invoked to explain these high-speed knotty ejecta.

He 2-111 (PK $\left.315-0^{\circ} 1\right)$ is an example of an extreme bipolar PN of large angular extent (11 arcmin across). The MES was used on the AAT to obtain high resolution long-slit spectra of 11 slit positions (Meaburn \& Walsh 1989) over the bright core and faint outer lobes. Extreme heliocentric radial velocities of $V_{\text {hel }}=-380 \mathrm{~km} \mathrm{~s}^{-1}$ and $+360 \mathrm{~km} \mathrm{~s}^{-1}$ are seen for the East and West lobes respectively implying hypersonic outflows generated by a nova-like process.

\section{References}

Meaburn, J., Blundell, B., Carling, R., Gregory, D. F., Keir, D., \& Wynne, C. G. 1984, MNRAS, 210, 463

Meaburn, J., \& Walsh, J.R. 1989, A\&A, 223, 277

Bryce, M., López, J. A., Holloway, A. J., \& Meaburn, J. 1997, ApJ, 487, L161

O’Connor, J. A., Redman, M. P., Holloway, A. J., Bryce, M., López, J. A., \& Meaburn, J. 2000, ApJ, 531, 336 